\title{
Observation of defect formation in metamaterials
}

\author{
Humeyra Caglayan, ${ }^{1}$ Irfan Bulu, ${ }^{2}$ Marko Loncar, ${ }^{2}$ and Ekmel Ozbay ${ }^{1}$ \\ ${ }^{1}$ Nanotechnology Research Center-NANOTAM, Department of Physics, Department of Electrical and \\ Electronics Engineering, Bilkent University, Bilkent, 06800 Ankara, Turkey \\ ${ }^{2}$ School of Engineering and Applied Sciences, Harvard University, 33 Oxford Street, Cambridge, \\ Massachusetts, 02138
}

We report subwavelength localization of electromagnetic fields within cavities based on metamaterials. Cavity resonances are observed in the transmission spectrum of a split ring resonator and composite metamaterials cavity structures. These cavity resonances are shown to exhibit high quality factors. Since the unit cells of metamaterials are much smaller than the operation wavelength, subwavelength localization is possible within these metamaterial cavity structures. In the present paper, we show that the electromagnetic field is localized into a region of $\lambda / 8$, where $\lambda$ is the cavity resonance wavelength.

In addition to the negative index of refraction, metamaterials have another important property: the unit cells of metamaterials are much smaller than the operating wavelength. This property can also lead to different novel applications, such as the localization of the field into a subwavelength region. Recently, the localization of the field has attained great interest from the scientific community, since it is the key issue of several applications. One method for obtaining a localized field is to make a deformation in a unit cell of the periodic structure. This phenomenon has been investigated experimentally and theoretically in photonic crystal structures. Photonic crystal defects have many important applications such as thresholdless semiconductor lasers and single-mode light-emitting diodes. However, defect structures in metamaterials are only investigated for periodically arranged single negative materials. To our knowledge, there has not been any experimental work conducted for the observation of cavities in left handed metamaterials. In the present letter, we investigate cavity formation in SRR and CMM structures. We first present the transmission results for SRR and CMM structures. Subsequently, we introduce the cavity structure and present the transmission of the SRR cavity and CMM cavity structures. Our results show that the modification of a unit cell of the metamaterial can exhibit a cavity resonance. Subsequently we show that the cavity formation in the metamaterials leads to the localization of the field within a subwavelength region. Finally, we present the effective group velocity reduction at the cavity resonances.

The SRR structure that we used for the present study was a one dimensional periodic arrangement of square rings. The CMM structure was obtained by the combination of the SRR structure and wire stripes. The structures were printed on a Teflon substrate with $\varepsilon=2.17$, in which the thickness of the substrate was $1 \mathrm{~mm}$. The wire stripes were on the back of the substrate and the square SRRs were on the front faces. The thickness of the copper was $0.05 \mathrm{~mm}$. The width of the wire stripes was $1.6 \mathrm{~mm}$. The lattice constant along the $\mathrm{x}$ direction (propagation direction) was $4.95 \mathrm{~mm}$. There were 5 layers along the propagation direction, in which the height of the structure was 40 layers. 30 layers of structures were stacked with a $2 \mathrm{~mm}$ period along the $\mathrm{z}$ direction. The E-field was in the y direction. The experimental setup consisted of an HP 8510C network analyzer and two standard gain horn antennae in order to measure the transmission 
amplitude. The calculations throughout the letter were performed by the commercial software program CST Microwave Studio.

The SRR structure that was used in this work has a band gap between 5-7 GHz. However, the closed ring resonator (CRR) structure, which was obtained by closing the splits in the rings, transmitted EM waves at these frequencies. Therefore, this gap is due to the magnetic resonance, in which the SRR structure exhibits a negative $\mu$ medium. When the electromagnetic field passes through the ring, an induced current is created and the generated field is perpendicular to the magnetic field of the light. The magnetic resonance results in a negative $\mu$. EM waves cannot propagate in the negative $\mu$ medium and possess a band gap in the spectrum. On the other hand, the CMM structure transmits EM waves at the band gap frequency of the SRR, since it has negative $\mu$ and $\varepsilon$ at this range.

In order to break the symmetry of these periodic left handed metamaterials, we changed the center unit cell of the structures by a closed ring structure, which were placed on both sides of the board and possessed positive $\varepsilon$ and $\mu$. This deformation in the SRR and CMM structures resulted in a cavity structure with a cavity resonance in the transmission spectrum. In Fig.1 (a) and (b) the transmission from the SRR cavity and CMM cavity structures are shown, respectively. The calculations agreed well with the experiments. We observed a cavity resonance with the Q-factor (quality factor, defined as the center frequency, divided by the full width at half maximum) of 192 at $6.7 \mathrm{GHz}(44.7 \mathrm{~mm})$ by the SRR cavity structure. The cavity resonance of the CMM structure was obtained at $7.5 \mathrm{GHz}(40 \mathrm{~mm})$ with a Q-factor of 108 .

The reflection of the SRR (CMM) structure is very high in the negative $\mu(\varepsilon)$ frequency range and, therefore, the SRR (CMM) structures on both sides of the cavity behave like frequencyspecific mirrors. Any propagating light that is trapped between them will bounce back and forth between these two mirrors. Since the mirrors localize light within a finite region; the modes are quantized into discrete frequencies, just as in Fabry-Perot resonances.
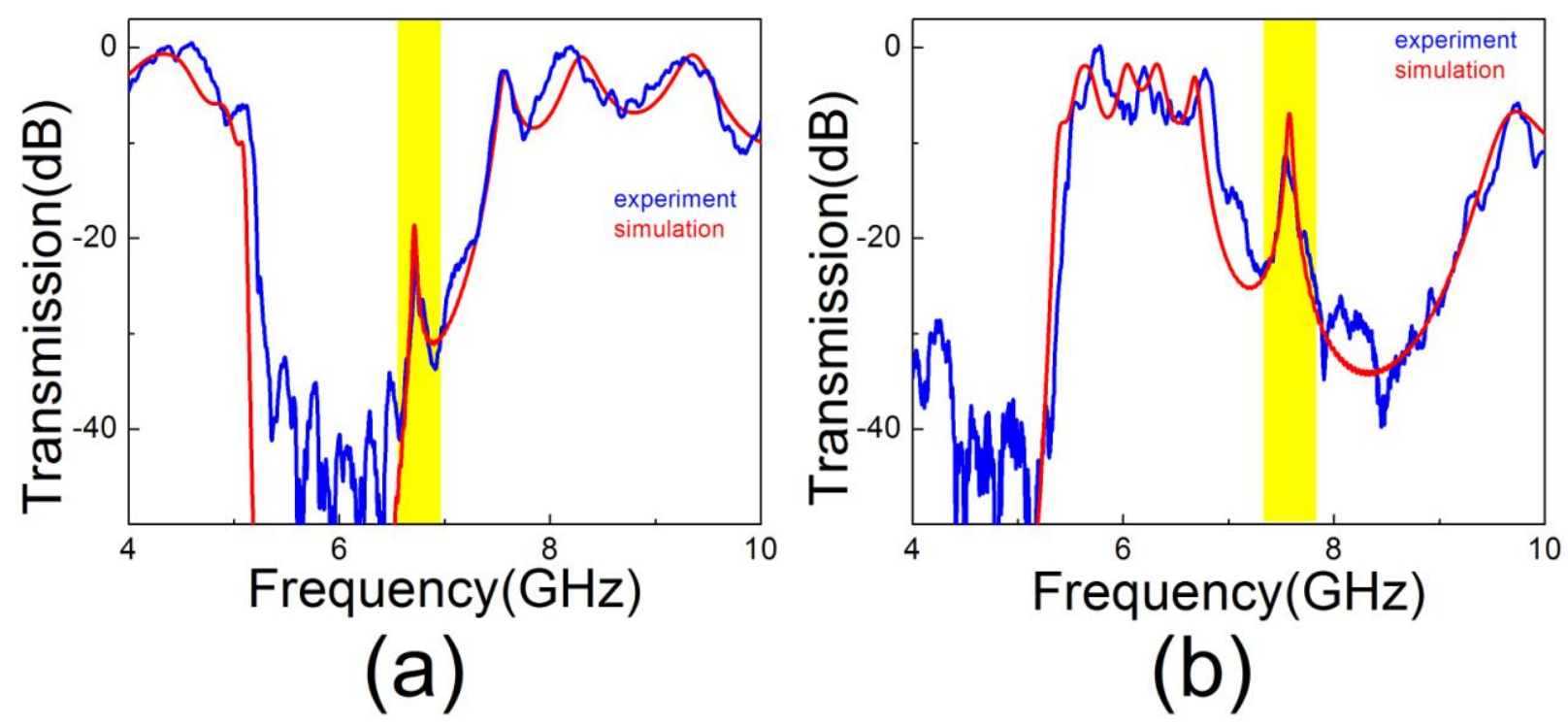

Fig.1 